\title{
Detection of influenza $A(\mathrm{H} 3 \mathrm{~N} 2)$ viruses exhibiting reduced susceptibility to the novel cap-dependent endonuclease inhibitor baloxavir in Japan, December 2018
}

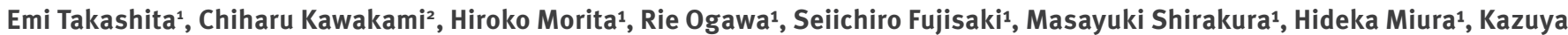
Nakamura $^{1}$, Noriko Kishida ${ }^{1}$, Tomoko Kuwahara ${ }^{1}$, Keiko Mitamura ${ }^{3}$, Takashi Abe ${ }^{4}$, Masataka Ichikawa5, Masahiko Yamazaki6, Shinji Watanabe ${ }^{1}$, Takato Odagiri ${ }^{1}$, on behalf of the Influenza Virus Surveillance Group of Japan ${ }^{7}$

1. Influenza Virus Research Center, National Institute of Infectious Diseases, Tokyo, Japan

2. Yokohama City Institute of Public Health, Kanagawa, Japan

3. Eiju General Hospital, Tokyo, Japan

4. Abe Children's Clinic, Kanagawa, Japan

5. Ichikawa Children's Clinic, Kanagawa, Japan

6. Zama Children's Clinic, Kanagawa, Japan

7. The members of the group are listed at the end of the article

Correspondence: Takato Odagiri (todagiri@nih.go.jp)

Citation style for this article:

Takashita Emi, Kawakami Chiharu, Morita Hiroko, Ogawa Rie, Fujisaki Seiichiro, Shirakura Masayuki, Miura Hideka, Nakamura Kazuya, Kishida Noriko, Kuwahara Tomoko, Mitamura Keiko, Abe Takashi, Ichikawa Masataka, Yamazaki Masahiko, Watanabe Shinji, Odagiri Takato, on behalf of the Influenza Virus Surveillance Group of Japan. Detection of influenza $\mathrm{A}\left(\mathrm{H}_{3} \mathrm{~N}_{2}\right)$ viruses exhibiting reduced susceptibility to the novel cap-dependent endonuclease inhibitor baloxavir in Japan, December 2018. Euro Surveill. 2019;24(3):pii=1800698. https://doi.org/10.2807/1560-7917.ES.2019.24.3.1800698

The novel cap-dependent endonuclease inhibitor baloxavir marboxil was approved for the treatment of influenza virus infection in Japan in February 2018. Two influenza $A\left(\mathrm{H}_{3} \mathrm{~N}_{2}\right)$ viruses carrying an I38T substitution in the polymerase acidic subunit $(\mathrm{PA})$ were detected in baloxavir-treated children in December 2018. This mutation is known to confer reduced susceptibility to baloxavir, and the two mutant viruses exhibited $76-$ and 120 -fold reduced susceptibility to baloxavir.

The novel antiviral drug baloxavir marboxil was approved in Japan on 23 February 2018 for the treatment of influenza virus infection, in patients 12 years and older and children younger than 12 years weighing $10 \mathrm{~kg}$ or more; it became available on 14 March 2018 in Japan (Figure). The hydrolysed active form of baloxavir marboxil (baloxavir acid) inhibits the cap-dependent endonuclease of influenza $A$ and $B$ viruses [1]. In Phase II and III clinical trials, I38T, I38F and I38M substitutions in the polymerase acidic subunit (PA) were detected in $A\left(\mathrm{H}_{1} \mathrm{~N}_{1}\right)$ pdmog and $A\left(\mathrm{H}_{3} \mathrm{~N}_{2}\right)$ influenza viruses $[2,3]$. Patients infected with these mutant viruses exhibited prolonged virus shedding, and the median time to symptom alleviation was longer in baloxavir recipients infected with viruses bearing these substitutions than in those infected with viruses that lacked these substitutions [2,3]. Therefore, we conducted nationwide monitoring of the baloxavir susceptibility of circulating influenza viruses by using a combination of phenotypic methods to analyse antiviral susceptibility and genotypic methods to detect amino acid substitutions [4].

\section{Detection of PA I38T mutant influenza $\mathrm{A}(\mathrm{H} 3 \mathrm{~N} 2)$ viruses}

In December 2018, influenza outbreaks occurred in two primary schools in Yokohama, Japan. We isolated four influenza $\mathrm{A}\left(\mathrm{H}_{3} \mathrm{~N}_{2}\right)$ viruses, $\mathrm{A} / \mathrm{YOKOHAMA} / 133 / 2018, \mathrm{~A} /$ YOKOHAMA/134/2018, A/YOKOHAMA/135/2018 and A/ YOKOHAMA/136/2018, from four children during these outbreaks (Table 1). Two patients aged 6 and 7 years, infected with $\mathrm{A} / \mathrm{YOKOHAMA} / 133 / 2018$ respectively $\mathrm{A} /$ YOKOHAMA/135/2018, were treated with a single oral dose of baloxavir marboxil. The third patient, 7-yearsold and infected with $A / Y O K O H A M A / 136 / 2018$, was treated with the neuraminidase (NA) inhibitor oseltamivir, whereas the last patient, aged 7 years and infected with A/YOKOHAMA/134/2018, had no exposure to antiviral drugs before specimen collection. Fever in the two children that received baloxavir resolved within 2 days of baloxavir administration, and in the child that received oseltamivir, it resolved within one day of oseltamivir administration. The child without treatment had a fever at the time of specimen collection.

Clinical specimens were collected 3 days after baloxavir administration, on day 2 of oseltamivir administration or on the day after onset. Sequencing analysis detected the PA I38T substitution in A/YOKOHAMA/133/2018 and $A / Y O K O H A M A / 135 / 2018$, but not in $A /$ 


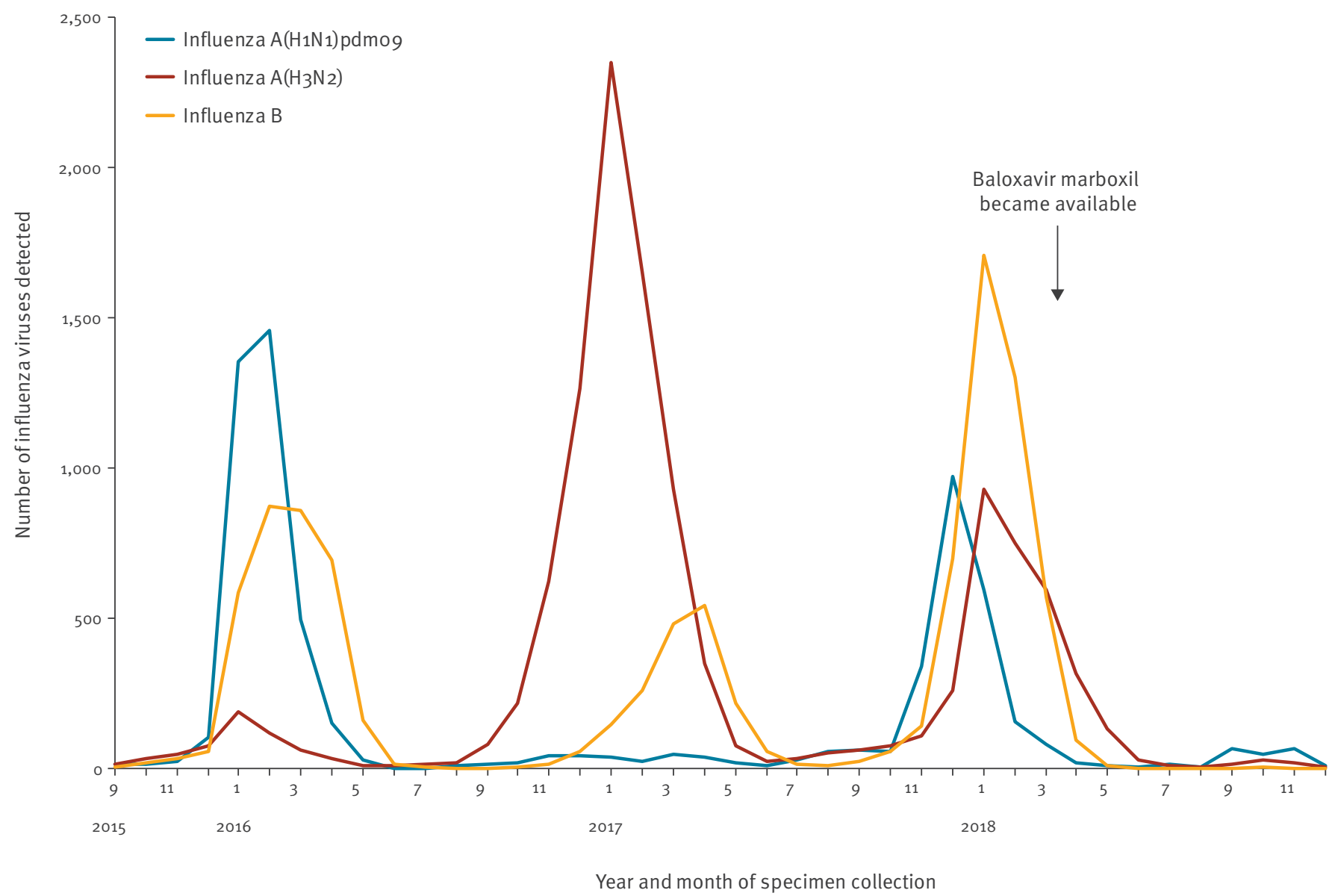

Monthly reports of influenza virus isolation/detection by the National Epidemiological Surveillance of Infectious Diseases. Baloxavir marboxil became available on 14 March 2018 in Japan.

YOKOHAMA/134/2018 or A/YOKOHAMA/136/2018. No amino acid substitutions associated with reduced susceptibility to NA inhibitors were detected. These results demonstrate that PA I38T mutant viruses were isolated from children 3 days after baloxavir administration.

\section{Antiviral susceptibilities of the PA I38T mutant viruses}

We compared the susceptibilities of the PA I38T mutant viruses and the wild-type virus to baloxavir and four NA inhibitors approved in Japan: oseltamivir, peramivir, zanamivir and laninamivir (Table 2). Antiviral susceptibilities were determined by using a focus reduction assay and a fluorescent NA inhibition assay with the NA-Fluor Influenza Neuraminidase Assay Kit (Applied Biosystems, California, United States) as previously described [4]. Baloxavir acid was purchased from MedChemexpress (New Jersey, United States). Oseltamivir carboxylate, peramivir and zanamivir were purchased from Sequoia Research Products
(Pangbourne, United Kingdom), and laninamivir was provided by Daiichi Sankyo Co., Ltd. (Tokyo, Japan). Results are expressed as the $50 \%$ inhibitory concentration $\left(\mathrm{IC}_{50}\right)$.

The $I C_{50}$ values of the viruses to baloxavir and the NA inhibitors are shown in Table 2. Both the PA I38T mutant viruses and the wild-type viruses showed normal inhibition with all four NA inhibitors, whereas the PA I38T mutant viruses exhibited 76 - and 120-fold higher IC ${ }_{50}$ values to baloxavir compared with the mean value of wild-type viruses. These results indicate that the PA I38T mutant viruses had reduced susceptibility to baloxavir, but remained susceptible to NA inhibitors.

\section{Discussion}

A Phase II clinical trial of baloxavir marboxil was conducted in Japan during the 2015/16 influenza season and Phase III trials were conducted in Japan and the United States in the $2016 / 17$ season $[2,3]$. The drug was approved in February 2018 in Japan and in 
TABLE 1

Influenza A(H3N2) viruses detected in outbreaks, Yokohama, Japan, December $2018(\mathrm{n}=4)$

\begin{tabular}{|c|c|c|c|c|c|}
\hline $\begin{array}{l}\text { GISAID } \\
\text { isolate ID }\end{array}$ & Isolate name & $\begin{array}{l}\text { Onset of } \\
\text { symptoms }\end{array}$ & $\begin{array}{l}\text { Antiviral } \\
\text { treatment }\end{array}$ & $\begin{array}{l}\text { Specimen } \\
\text { collection }\end{array}$ & $\begin{array}{c}\text { PA } \\
\text { substitution }\end{array}$ \\
\hline EPI_ISL_332908 & A/YOKOHAMA/133/2018 & 2 Dec 2018 & $\begin{array}{c}3 \text { Dec } 2018 \\
\text { Baloxavir }\end{array}$ & 6 Dec 2018 & I38T (277Y) \\
\hline EPI_ISL_332910 & A/YOKOHAMA/135/2018 & 4 Dec 2018 & $\begin{array}{l}4 \text { Dec } 2018 \\
\text { Baloxavir }\end{array}$ & 7 Dec 2018 & 138T (Y277F) \\
\hline EPI_ISL_332911 & A/YOКОНАМА/136/2018 & 4 Dec 2018 & $\begin{array}{c}\text { 5-9 Dec } 2018 \\
\text { Oseltamivir }\end{array}$ & 7 Dec 2018 & $381(277 Y)$ \\
\hline EPI_ISL_332909 & A/YOKOHAMA/134/2018 & 6 Dec2018 & None & 7 Dec 2018 & $38 \mathrm{I}(\mathrm{Y} 277 \mathrm{~F})$ \\
\hline
\end{tabular}

GISAID: Global Initiative on Sharing All Influenza Data; PA: polymerase acidic subunit.

aThe amino acid residue at position 277 is not involved in PA-inhibitor interactions.

TABLE 2

Susceptibility of influenza A(H3N2) viruses detected in outbreaks, Yokohama, Japan, December $2018(\mathrm{n}=4)$

\begin{tabular}{|c|c|c|c|c|c|c|}
\hline \multirow{3}{*}{ Isolate name } & \multirow{3}{*}{$\begin{array}{c}\text { PA } \\
\text { substitution }\end{array}$} & \multicolumn{5}{|c|}{$\mathrm{I} \mathrm{C}_{5} \mathrm{O}, \mathrm{nM}$} \\
\hline & & \multirow{2}{*}{ Baloxavir } & \multicolumn{4}{|c|}{ Neuraminidase inhibitors (WHO criteria) } \\
\hline & & & Oseltamivir ${ }^{\mathrm{a}}$ & Peramivir ${ }^{\mathrm{a}}$ & Zanamivir $^{\mathrm{a}}$ & Laninamivira \\
\hline A/YOKOHAMA/133/2018 & I38T & 227.08 & $0.41(\mathrm{NI})$ & $0.16(\mathrm{NI})$ & $0.88(\mathrm{NI})$ & $0.76(\mathrm{NI})$ \\
\hline A/YOKOHAMA/135/2018 & I38T & 144.02 & $0.25(\mathrm{NI})$ & $0.15(\mathrm{NI})$ & $0.81(\mathrm{NI})$ & $0.92(\mathrm{NI})$ \\
\hline A/YOKOHAMA/136/2018 & 381 & 2.78 & $0.38(\mathrm{NI})$ & $0.12(\mathrm{NI})$ & $0.77(\mathrm{NI})$ & $1.05(\mathrm{NI})$ \\
\hline A/YOKOHAMA/134/2018 & 381 & 1.02 & $0.28(\mathrm{NI})$ & $0.12(\mathrm{NI})$ & $0.76(\mathrm{NI})$ & $0.88(\mathrm{NI})$ \\
\hline
\end{tabular}

PA: polymerase acidic subunit; IC $: 50 \%$ inhibitory concentration; NI: Normal inhibition; WHO: World Health Organization.

The median IC values of 30 influenza $A\left(\mathrm{H}_{3} \mathrm{~N}_{2}\right)$ viruses isolated in the $2018 / 19$ influenza season in Japan to oseltamivir, peramivir, zanamivir and laninamivir were $0.22 \pm 0.08,0.11 \pm 0.02,0.50 \pm 0.26$ and $0.98 \pm 0.26$, respectively.

October 2018 in the United States. During the 2015/16 and 2016/17 seasons, influenza $\mathrm{A}\left(\mathrm{H}_{1} \mathrm{~N}_{1}\right)$ pdmog and $A\left(\mathrm{H}_{3} \mathrm{~N}_{2}\right)$ viruses, respectively, predominated in Japan. During the Phase II trial, the PA 138T and 138F substitutions emerged after baloxavir treatment in four (3.6\%) of $112 \mathrm{~A}\left(\mathrm{H}_{1} \mathrm{~N}_{1}\right)$ pdmog viruses isolated from adults aged 20-64 years [4]. In the Phase III trials, the PA I38T and I38M substitutions emerged in 36 (9.7\%) of $370 \mathrm{~A}\left(\mathrm{H}_{3} \mathrm{~N}_{2}\right)$ viruses obtained from patients aged $12-64$ years and in $18(23.4 \%)$ of $77 \mathrm{~A}\left(\mathrm{H}_{3} \mathrm{~N}_{2}\right)$ viruses obtained from children aged 6 months to<12 years $[2,3]$. Cumulative data from clinical trials of oseltamivir, involving almost 2,000 oseltamivir-treated patients, indicate that the incidence of reduced susceptibility to oseltamivir is $0.32 \%$ in adults and $4.1 \%$ in children (if low-level mutants detected by genotyping alone in mixed virus populations are included, then the corresponding values are $0.4 \%$ and $5.4 \%$, respectively) [5]. These results suggest that the incidence of influenza viruses exhibiting reduced susceptibility to baloxavir is higher than that to oseltamivir.
In Japan, baloxavir marboxil became available at the end of the 2017/18 influenza season. We isolated two PA $138 \mathrm{~T}$ mutant influenza $\mathrm{A}\left(\mathrm{H}_{3} \mathrm{~N}_{2}\right)$ viruses from baloxavir-treated children in December 2018. PA 138 is highly conserved in influenza $A$ and $B$ viruses [3], and the I38T substitution was not detected among 17,227 PA sequences from $A\left(\mathrm{H}_{3} \mathrm{~N}_{2}\right)$ viruses in the National Institute of Allergy and Infectious Diseases (NIAID) Influenza Research Database (IRD) [6]. Furthermore, our sequencing analysis revealed that these two PA I38T mutant viruses possessed different PA sequences and thus originated from different viruses, suggesting no human-to-human transmission. Our findings indicate that these viruses emerged under the selective pressure of baloxavir marboxil. In contrast, no viruses exhibiting reduced susceptibility to NA inhibitors were detected among 90 influenza A viruses tested between September and December 2018 in Japan [7]. These observations suggest that the emergence of PA 138T mutant viruses may increase as the use of baloxavir marboxil increases in the 2018/19 influenza season. Therefore, the baloxavir susceptibility of influenza viruses should be closely monitored. 
In vitro studies using the plaque reduction assay revealed that influenza $A / W S N / 33\left(\mathrm{H}_{1} \mathrm{~N}_{1}\right)$ viruses with the PA I38T or I38F substitutions show 27.2- and 10.6fold higher $\mathrm{EC}_{50}$ values (the $50 \%$ effective concentration) to baloxavir compared with the wild-type virus [3]. Furthermore, influenza A/Victoria/3/75( $\left.\mathrm{H}_{3} \mathrm{~N}_{2}\right)$ viruses with the PA $138 \mathrm{~T}$ or $138 \mathrm{M}$ substitutions showed 56.6and 13.8-fold higher $\mathrm{EC}_{50}$ values, respectively [3]. These results suggest that the PA $138 \mathrm{~T}$ substitution has a marked impact on baloxavir susceptibility. In the present study, we obtained two influenza $A\left(\mathrm{H}_{3} \mathrm{~N}_{2}\right)$ clinical isolates possessing the PA 138T substitution. These viruses showed 76- and 120 -fold higher $I C_{50}$ values to baloxavir compared with the mean value of wild-type viruses. Our data thus demonstrate that the PA I38T substitution is associated with reduced susceptibility to baloxavir in currently circulating influenza $A\left(\mathrm{H}_{3} \mathrm{~N}_{2}\right)$ viruses.

The Technical Expert Working Group of the World Health Organization's (WHO) Global Influenza Surveillance and Response System (GISRS) for Surveillance on Antiviral Susceptibility (WHO-AVWG) has established a set of criteria to define the NA inhibitor susceptibility of influenza viruses based on the fold-change in $I_{50}$ value compared with the median value for viruses from the same type/subtype/lineage [8]. For influenza A virus, use of the terms normal ( $<10$-fold increase), reduced (10-100-fold increase) and highly reduced (>100-fold increase) inhibition is recommended when reporting and analysing surveillance data; for influenza $B$, the same definitions are used but for $<5$-fold, 5-50-fold and $>50$-fold increases. The WHO-AVWG is currently collecting more data on baloxavir marboxil to establish a similar set of criteria to define baloxavir susceptibility, which should be available in the near future.

In summary, our results indicate that continuous monitoring of the emergence of baloxavir-resistant viruses is important for public health planning and clinical recommendations for antiviral drug use.

\section{Acknowledgements}

We thank Miki Akimoto, Aya Sato, Hiromi Sugawara, and Kayo Watanabe for technical assistance. We also thank Misaki Hyodo, Eriko Mizukami, Sawako Kawahara, and Minako Imae (Yokohama City Public Health Center) for providing clinical specimens, Kohei Shimizu and Hiroki Ozawa (Yokohama City Institute of Public Health) for isolating influenza viruses, and Susan Watson for scientific editing.

\section{The Influenza Virus Surveillance Group of Japan:}

The members of the Influenza Virus Surveillance Group of Japan are: Rika Komagome (Hokkaido Institute of Public Health), Asami Ohnishi (Sapporo City Institute of Public Health), Rika Tsutsui (Aomori Prefectural Public Health and Environment Center), Masaki Takahashi (Iwate Prefectural Research Institute for Environmental Sciences and Public Health), Mie Sasaki (Miyagi Prefectural Institute of Public Health and Environment), Shiho Tamura (Sendai City Institute of Public Health), Chihiro Shibata (Akita Prefectural Research Center for Public Health and Environment), Shizuka Tanaka
(Yamagata Prefectural Institute of Public Health), Nozomi Saito (Fukushima Prefectural Institute of Public Health), Chika Hirokawa (Niigata Prefectural Institute of Public Health and Environmental Sciences), Kazunari Yamamoto (Niigata City Institute of Public Health and Environment), Aoi Saito (Ibaraki Prefectural Institute of Public Health), Izumi Kushibuchi (Tochigi Prefectural Institute of Public Health and Environmental Sciences), Shunsuke Kataoka (Utsunomiya City Institute of Public Health and Environment Science), Hiroyuki Tsukagoshi (Gunma Prefectural Institute of Public Health and Environmental Sciences), Noriko Suzuki (Saitama Institute of Public Health), Yuka Uno (Saitama City Institute of Health Science and Research), Hiroyuki Hashimoto (Chiba Prefectural Institute of Public Health), Wakako Nishikawa (Chiba City Institute of Health and Environment), Mami Nagashima (Tokyo Metropolitan Institute of Public Health), Sumi Watanabe (Kanagawa Prefectural Institute of Public Health), Hideaki Shimizu (Kawasaki City Institute of Public Health), Hazime Amano (Yokosuka Institute of Public Health), Sayoko Arakawa (Sagamihara City Institute of Public Health), Masayuki Oonuma (Yamanashi Institute for Public Health), Michiko Takeuchi (Nagano Environmental Conservation Research Institute), Yuichiro Okamura (Nagano City Health Center), Yukiko Sakai (Shizuoka Institute of Environment and Hygiene), Takaharu Maehata (Shizuoka City Institute of Environmental Sciences and Public Health), Toshihiko Furuta (Hamamatsu City Health Environment Research Center), Tetsuya Yoneda (Toyama Institute of Health), Hiroe Kodama (Ishikawa Prefectural Institute of Public Health and Environmental science), Kaori Sato (Fukui Prefectural Institute of Public Health and Environmental Science), Masahiro Nishioka (Gifu Prefectural Research Institute for Health and Environmental Sciences), Yusuke Sato (Gifu Municipal Institute of Public Health), Yoshihiro Yasui (Aichi Prefectural Institute of Public Health), Takuya Yano (Mie Prefecture Health and Environment Research Institute), Asa Tanino (Shiga Prefectural Institute of Public Health), Sachi Hirata (Kyoto Prefectural Institute of Public Health and Environment), Akiko Nagasao (Kyoto City Institute of Health and Environmental Sciences), Satoshi Hiroi and Hideyuki Kubo (Osaka Institute of Public Health), Fumika Okayama (Sakai City Institute of Public Health), Tomohiro Oshibe (Hyogo Prefectural Institute of Public Health and Consumer Sciences), Ai Mori (Kobe Institute of Health), Ryutaro Murayama (Amagasaki City Institute of Public Health), Misako Fujitani (Nara Prefecture Institute of Health), Yuki Matsui (Wakayama Prefectural Research Center of Environment and Public Health), Takashi Nishiyama (Wakayama City Institute of Public Health), Koji Takeuchi (Tottori Prefectural Institute of Public Health and Environmental Science), Tetsuo Mita (Shimane Prefectural Institute of Public Health and Environmental Science), Kayoko Nomiya (Okayama Prefectural Institute for Environmental Science and Public Health), Yukie Shimazu (Hiroshima Prefectural Technology Research Institute), Yoshiki Fujii (Hiroshima City Institute of Public Health), Shoichi Toda (Yamaguchi Prefectural Institute of Public Health and Environment), Yumiko Kawakami (Tokushima Prefectural Public Health, Pharmaceutical and Environmental Sciences Center), Yukari Terajima (Kagawa Prefectural Research Institute for Environmental Sciences and Public Health), Mayumi Yamashita (Ehime Prefecture Institute of Public Health and Environmental Science), Tomiyo Takahashi (Public Health Institute of Kochi Prefecture), Yuki Ashizuka (Fukuoka Institute of Health and Environmental Sciences), Chinami Wasano (Fukuoka City Institute of Health and Environment), Takashi Kimura (Kitakyushu City Institute of Health and Environmental Sciences), Katsuyuki Ando (Saga Prefectural Institute of Public Health and Pharmaceutical Research), Ayaka Yamashita (Nagasaki Prefectural Institute for Environment Research and Public Health), Takashi Sakai (Kumamoto Prefectural Institute of Public-Health and Environmental Science), Kaori Nishizawa (Kumamoto City Environmental Research Center), Toru Hayashi (Oita Prefectural Institute of Health and Environment), Miho 
Miura (Miyazaki Prefectural Institute for Public Health and Environment), Yuka Hamada (Kagoshima Prefectural Institute for Environmental Research and Public Health), and Yumani Kuba (Okinawa Prefectural Institute of Health and Environment).

Funding statement: This work was supported by a Grant-inAid for Emerging and Reemerging Infectious Diseases from the Ministry of Health, Labour and Welfare, Japan (grant no. 10110400) and by JSPS KAKENHI Grant number 18K10036.

\section{Conflict of interest}

None declared.

\section{Authors' contributions}

Designed the analyses: ET, SW, TO. Analysed and interpreted the data: ET, CK, HM, RO, SF, MS, HM, KN, NK, TK, KM, TA, MI, MY, SW, TO. Drafted the article: ET. Revised the article: SW, TO.

\section{References}

1. Koszalka P, Tilmanis D, Hurt AC. Influenza antivirals currently in late-phase clinical trial. Influenza Other Respir Viruses. 2017;11(3):240-6. https://doi.org/10.1111/irv.12446 PMID: 28146320

2. Hayden FG, Sugaya N, Hirotsu N, Lee N, de Jong MD, Hurt AC, et al. Baloxavir marboxil for uncomplicated influenza in adults and adolescents. N Engl J Med. 2018;379(10):913-23. https:// doi.org/10.1056/NEJMoa1716197 PMID: 30184455

3. Omoto S, Speranzini V, Hashimoto T, Noshi T, Yamaguchi H, Kawai $M$, et al. Characterization of influenza virus variants induced by treatment with the endonuclease inhibitor baloxavir marboxil. Sci Rep. 2018;8(1):9633. https://doi.org/10.1038/ S41598-018-27890-4 PMID: 29941893

4. Takashita E, Morita H, Ogawa R, Nakamura K, Fujisaki S, Shirakura M, et al. Susceptibility of influenza viruses to the novel cap-dependent endonuclease inhibitor baloxavir marboxil. Front Microbiol. 2018;9(3026):3026. https://doi. org/10.3389/fmicb.2018.03026 PMID: 30574137

5. Aoki FY, Boivin G, Roberts N. Influenza virus susceptibility and resistance to oseltamivir. Antivir Ther. 2007;12(4 Pt B):603-16. PMID: 17944268

6. Influenza Research Database (IRD). Analyze Sequence Variation (SNP). Bethesda: National Institute of Allergy and Infectious Diseases. [Accessed: 15 Jan 2019]. Available from: https://www.fludb.org

7. National Institute of Infectious Diseases (NID). Antiviral resistance surveillance in Japan. Tokyo: NID. [Accessed: 15 Jan 2019]. Available from: http://www.nih.go.jp/niid/en/influresist-e.html

8. World Health Organization. Meetings of the WHO working group on surveillance of influenza antiviral susceptibility Geneva, November 2011 and June 2012. Wkly Epidemiol Rec. 2012;87(39):369-74. PMID: 23061103

\section{License and copyright}

This is an open-access article distributed under the terms of the Creative Commons Attribution (CC BY 4.0) Licence. You may share and adapt the material, but must give appropriate credit to the source, provide a link to the licence, and indicate if changes were made.

This article is copyright of the authors or their affiliated institutions, 2019. 